\title{
An Analysis of the Global and Partial Voice Handicap Index in Patients with Early Glottic Carcinoma Treated with High Level of Irradiation
}

\author{
Daniela Musio ${ }^{1}$, Francesca De Felice ${ }^{1}$, Cristina Bigelli ${ }^{2}$, Nadia Bulzonetti ${ }^{1}$, Roberta Guarnaccia ${ }^{1}$, \\ Mario Tombolini ${ }^{2}$, Giovanni Ruoppolo ${ }^{2}$, Marco de Vincentiis ${ }^{2}$, Vincenzo Tombolini ${ }^{1}$ \\ ${ }^{1}$ Dipartimento di Scienze Radiologiche Oncologiche e Anatomo-Patologiche, Catterdra di Radioterapia, Università degli Studi di \\ Roma "Sapienza”, Roma, Italy; ${ }^{2}$ Dipartimento di Otorinolaringoiatria, Audiologia e Foniatria “Giorgio Ferreri”, Università degli \\ Studi di Roma "Sapienza”, Roma, Italy. \\ Email: daniela.musio@libero.it
}

Received November $17^{\text {th }}, 2013$; revised December $15^{\text {th }}, 2013$; accepted December $23^{\text {rd }}, 2013$

Copyright (C) 2014 Daniela Musio et al. This is an open access article distributed under the Creative Commons Attribution License, which permits unrestricted use, distribution, and reproduction in any medium, provided the original work is properly cited. In accordance of the Creative Commons Attribution License all Copyrights (C) 2014 are reserved for SCIRP and the owner of the intellectual property Daniela Musio et al. All Copyright (C) 2014 are guarded by law and by SCIRP as a guardian.

\section{ABSTRACT}

Background: This is a retrospective study on a group of patients with early glottic carcinoma, treated with curative radiotherapy. The aim of the study is to assess voice quality after treatment. We also evaluated local recurrence, overall survival, disease free survival and toxicity. Material and Methods: We examined a total of 36 patients, out of which 27 were smokers: 10 females/5 smokers; 26 males/22 smokers. The sample was observed from January 2007 to July 2012 (average follow-up period: 33.5 months, range 12 - 76). Diagnosis of early glottic carcinoma was eight during the two-year period 2007/2008, nine during 2009/2010, eleven during year 2011 and eight during year 2012. All patients suffering from early glottic carcinoma, histopathologically confirmed, were classified as follows: 24 patients T1a, 3 patients T1b, 6 patients T2 and 3 patients had a carcinoma in situ. They were treated only with conventional radiotherapy. After the treatment (total dose $70 \mathrm{~Gy}$ ), patients were asked to fill out the Voice Handicap Index (VHI) questionnaire, composed of 30 questions that covered physical, emotional and functional areas. Results: The global VHI was evidenced with good voice quality $(62 \%$ of patients: VHI < 30; 34\% of patients: VHI 31 - 60). Vocal disability was mild in over $70 \%$ of patients within the physical area and over $80 \%$ within the emotional area. We observed two local recurrences, one occurred five years after the end of radiation therapy and one six months after. In terms of toxicity, we observed that acute reactions, such as dysphonia, dysphagia and erythema, were relatively limited. Conclusions: The VHI index suggests that radiotherapy produces acceptable functional results, with limited repercussion on life quality. In line with literature, our data confirmed good cure rates and larynx preservation (94.4\%).

\section{KEYWORDS}

\section{Glottic Carcinoma; Voice Handicap Index; Radiotherapy}

\section{Introduction}

Larynx cancer is the most common malignancy of the upper aerodigestive tract. In the United States, during 2013, the estimated number of new cases of larynx cancer was about $0.8 \%$ (12.260) of all new cancer cases between both sexes. In the majority of cases, it was associated with smoke and prevailed among men. In fact, 79\% of new cases occur among men, and the same percent- age applies to estimated deaths [1].

The treatment aim, for early invasive larynx carcinoma, is to obtain cure with laryngeal preservation associated with optimal voice quality and minimal morbidity [2]. At the moment, early stage cancer, classified as in situ carcinomas, T1 and T2, can effectively be treated with both radiation and surgery. The treatment of choice still remains controversial as there is insufficient evidence to establish 
one modality as superior to the other. There are no randomized trials comparing radiotherapy versus surgeries and the only randomized controlled trials comparing the two methodologies are limited to the reporting of study design and analysis [3]. In general, the rates of local control, laryngeal voice preservation and survival are comparable for patients treated with radiotherapy or transoral laser excision. If radiotherapy fails, salvage surgery is successful in $75 \%$ to $85 \%$ of the patients. In literature, local control rate is from $67 \%$ to $96 \%$ and larynx is preserved in $85 \%$ - 97\% of irradiated patients [4]. Usually, researches value effective treatment in terms of overall survival and disease free survival, with regard to toxic side effects, provable for a cost-effective valuation [59].

When different treatment strategies offer similar cure rates, resulting voice quality becomes paramount in the therapeutic decision. Quality of voice is recognized as an important endpoint, such as disease free survival and overall survival [10]. However, it is important to realize that the meaning of "voice quality" is still unclear and takes shades of meaning for each patient, his experience and his expectation. The voice quality is often said to be "functionally" normal, usually without a definition of functionality being given.

Mlynarek et al. [9] compared surgery and radiotherapy in terms of voice outcome in a retrospective comparative review of 36 patients with T1-2 glottic carcinoma and concluded that surgery tends to result in a better voice outcome.

We felt the need to value voice quality in patients after radiotherapy for laryngeal cancer. Frequently patients feel hopeless when receiving a diagnosis of larynx cancer, believing that treatment is necessarily related to voice loss, which is so important for social interactions. Several studies took care of voice quality problems following treatment of early glottic cancer, but results are contradictory [11-17]. The aim of this work was to quantify vocal disability and to realize if a worsening of voice quality resulted in limitation in social life. We evaluated local recurrence, overall survival, disease free survival and acute and late toxicity.

\section{Methods and Materials}

Patient selection. This is a single-Institute clinical study (Policlinico Umberto I, "Sapienza” University of Rome). Inclusion criteria were: early glottic carcinoma treated with radiation; a follow-up period of at least 12 months after the end of radiotherapy; no evidence of metastatic disease and no history of radiotherapy; absence of recently treated illnesses and/or other diseases interfering with either vocal function or general health at the time of inclusion; alive and still in follow-up program. Patients included in the sample had histologically confirmed early glottic squamous cell carcinoma and were staged, based on data in their charts, according to clinical classification of the American Joint Committee on Cancer AJCC (7th ED., 2010). Patients singed an informed consent.

Treatment plan. All patients underwent nasopharyngolaryngoscopy, biopsy and complete blood count. Imaging included thin-cut CT and/or MRI of the head and neck and a chest X-ray. Preventive dental care occurred 15 days before radiotherapy and nutrition evaluations too. Patients were assessed for toxicity weekly and underwent weekly complete blood counts. They were treated supine and were immobilized in a thermoplastic shell, fixed to the couch in at least three places. Shoulders were positioned as inferiorly as possible, permitting lateral radiation beams to treat the larynx without the need to angle them. CT scan was performed with $5 \mathrm{~mm}$ slices obtained from base of skull to the top of the aortic arch. CT data were sent to the Treatment Planning Software (Pinnacle ${ }^{\circledR}$ ) for target volume definition and dose solutions. The planning target volume (PTV) encompassed the glottic larynx. Superior and inferior field border were inferior margin of hyoid bone and inferior margin of cricoid cartilage, respectively. Anterior field border was $1 \mathrm{~cm}$ to the skin and posterior border was the anterior edge of the vertebral body. Radiotherapy dose given was 70 Gy in 35 daily fractions, for 7 weeks, using a $6 \mathrm{MV}$ linear accelerator. We used electronic portal imaging to verify treatment set-up; portal images, taken weekly, were compared with DRRs from the planning CT scan.

Voice handicap index analysis. Patients were surveyed 12 - 13 months after the end of radiation therapy using a validated questionnaire, The Voice Handicap Index (VHI) proposed by Jacobson et al. [2], and used in several assessments that can be found in recent literature [4,8,11-14,17-20].

The questionnaire comprises 30 items that allow to evaluate voice quality and its influence on patient's psychology and on his social life. Questions list is reported in Table 1.

The items are grouped in three sections that relate to physical, emotional and functional area (10 questions for each area). Possible answers were the following: never (0), almost never (1), sometimes (2), almost always (3), always (4). The multi-item scale scores were converted linearly to a 0 - 120 scale, with higher score representing worse vocal disability: mild (VHI < 30), moderate (VHI 31 - 60), severe (VHI 61 - 90) and very severe (91 - 120). The same criteria can be applied to evaluate each area of the VHI (mild: VHI < 10, moderate: VHI 11 - 20, severe: VHI 21 - 30, and very severe: VHI 31 - 40).

The methodology applied provides high data-quality standards and has produced a positive effect in terms of 
Table 1. Statement of questionnaire.

Physical domain
P1-My voice makes it difficult for people to hear me
P2-People have difficulty understanding me in a noisy room
P3-People ask me to repeat myself when speaking face-to-face
P4-My family has difficulty hearing me when I call them throughout
the house
P5-I use the phone less often than I would like
P6-I tend to avoid groups of people because of my voice

P7-I speak whit friends, neighbors, or relatives less often because of my voice

P8-My voice difficulties restrict my personal and social life

P9-I feel left out of conversations because of my voice

P10-My voice problem causes me to lose income

Emotional domain

E1-I'm tense when talking with others because of my voice

E2-People seem irritated with my voice

E3-I find other people don’t understand my voice problem

E4-My voice problem upset me

E5-I'm less out-going because of my voice problem

E6-My voice makes me feel handicapped

E7-I feel annoyed when people ask me to repeat

E8-I feel embarrassed when people ask me to repeat

E9-My voice makes me feel incompetent

E10-I'm ashamed of my voice problem

\section{Functional domain}

F1-I run out of air when I talk

F2-The sound of my voice varies throughout the day

F3-People ask "what's wrong with your voice?”

F4-My voice sounds creaky and dry

F5-I feel as though I have to strain to produce voice

F6-The clarity of my voice is unpredictable

F7-I try to change my voice to sound different

F8-I use a great deal of effort to speak

F9-My voice is worse in the evening

F10-My voice "gives out" on me in the middle of speaking

relation with the patient. We clarified all questions to patients to avoid any possible mis-interpretation, and specified the meaning of each score, to prevent (or at least limit) wrong score attribution. The time of completion has been, on average, 10 minutes. Therefore, time for completion can be fit within the therapy control protocol.

Data and statistical analysis. Overall survival and disease-free survival were considered in months from the end of the treatment. Standard descriptive statistics were used to evaluate the distribution of each answer. Univariate analysis, using Fisher's exact test, was performed to determine statistical significance.

\section{Results}

Patient characteristics. We observed a total of 36 patients: 10 females and 26 men; the patients' age range was 43 - 86 years (average 66.75); 27 patients were smokers (22 males/5 females). On a total of 36 cases of early glottic carcinoma, 3 patients were diagnosed with carcinoma in situ, 27 patients with T1 squamous cell of the vocal cord (of these 3 patients were T1b), 6 patients with T2 glottic carcinoma. All patients were fully active or restricted only in physically strenuous activity (ECOG performance status 0 - 1).

Toxicity and survival. Severe acute toxicity were not recorded. Dysphagia was the most common symptom and the earliest manifestation of acute toxicity. Grade 1 or 2 acute toxic effects that occurred among patients are summarized in Table 2. As of July 2013, surviving patients had been monitored for an average period of 33.5 months (range, 12 - 76). Out of these 36 patients, 22.2\% were followed for at least five years, $5.6 \%$ for at least four years, and $19.4 \%$ for at least three years. No deaths occurred during follow-up. Local recurrence occurred in two patients: both patients underwent surgery, requiring total laryngectomy. None had distant recurrences. The overall survival was $100 \%$. The rate of disease-free survival was $94.4 \%$.

Voice handicap index. Patients underwent subjective voice assessment. To quantify patient opinion regarding the impact caused by radiotherapy, the Voice Handicap

Table 2. Acute toxicity.

\begin{tabular}{cccc}
\hline \multirow{2}{*}{ Week } & \multicolumn{3}{c}{ Toxicity effects } \\
\cline { 2 - 4 } & Dysphagia & Dysphonia & Erythema \\
\hline I & 0 & 0 & 0 \\
II & 4 & 1 & 0 \\
III & 18 & 2 & 0 \\
IV & 1 & 1 & 0 \\
V & 2 & 4 & 6 \\
VI & 2 & 1 & 7 \\
VII & 0 & 2 & 2 \\
\hline
\end{tabular}


Index was employed. 29 of the 36 patients completed the VHI questionnaire. VHI index was developed specifically to correlate how much voice change may affect the patient's quality of life, considering in detail physical, emotional and functional aspects.

Preliminary data analysis has evidenced consistent answers. Within each of the three areas there is a concentration on the answer "never".

In the physical area, the answer "never" to questions P3, P5, P6, P7, P8 has uniform frequency between 70 and $80 \%$; frequency in answer "almost always" is higher than answer "sometimes" (except for questions P3 and P8). In the emotional area, all answers have same frequency, at a level of $70 \%-80 \%$ except for questions E4 and E10. In the functional area, data are more homogeneous: answer "never" is significantly more frequent than the others (about twice, at a level of $50 \%$ ) in questions F6, F7, F8, F9, F10; in questions F4 the answer "sometimes" is the most frequent (34\%). Frequency range, for each area, are reported in Table 3.

Table 4 summarizes results of our analysis, reporting VHI for physical, emotional and functional areas and global VHI, for each patient. Among the total answers, average VHI was 24.34, with a standard deviation of 15.77, with exclusion of one outlier data; VHI and standard deviation for the three areas were the following: 6.41/6.82 in the physical area, 4.83/7.14 in the emotional area, 13.10/8.73 in the functional area. The comparison of final results among the groups (global vs area) does not show significant differences.

Vocal disability was mild in over $70 \%$ of the patients, within the physical area and in over $80 \%$ of the patients within the emotional area. Within the functional area, vocal disability resulted mild in $35 \%$ of the patients and moderate for $35 \%$ of the patients. The global VHI underlined good voice quality (62\% of patients: VHI < 30; $34 \%$ of patients: VHI $31-60$ ).

\section{Discussion}

The patients' age is similar to other published studies, and therefore highlights that malignant laryngeal tumors are more frequent in the sixth and seventh decades of life $[8,13,21]$. Moreover, it is estimated that about $75 \%$ of larynx cancer are attributable to cigarette smoking as we show in our data.

Concerning data acquisition, more synthetic questionnaires can be found in literature, such us a questionnaire [4] made of the following 5 questions: Does your voice sound deviant, e.g. breathy or rough?; Do you encounter problems holding conversation due to your voice?; Do you encounter problems making a telephone call due to your voice?; Do you encounter problems shouting?; Do you have to strain to produce voice?

Dutch experiences favours the 5 -item questionnaire for four main reasons: 1 ) the questions are simple, 2) patient do not need help completing the questions, 3) completion of the questionnaire takes less than 2 min, and 4) interpretation of the results is straightforward. From a statistical point of view, reliability (assessed by test-retest intraclass correlation coefficients), internal consistency, interrelation (assessed by Cronbach's alpha, and Spearman correlation coefficients), and predictive validity ( $p$ values) proved to be good.

In our study, we selected the extensive questionnaire to allow results' comparison with our previous researches [19], to include emotional components in post-treatment assessment, relevant for patients' quality of life, and to obtain a more detailed picture of each patient's overall heath.

Voice quality after radiation for larynx cancer is typically very good and superior to surgery and improves a few months after irradiation [11,22].

There are few papers in literature assessing voice after radiotherapy or laser surgery, and only rarely is there a comparison with a control group of normal individuals

Table 3. Frequency range.

\begin{tabular}{|c|c|c|c|c|c|c|}
\hline & & \multicolumn{5}{|c|}{ Answer } \\
\hline & & 0 & 1 & 2 & 3 & 4 \\
\hline \multirow{2}{*}{$\mathrm{P}$} & Min & P2: $45 \%$ & P10: 0\% & P10: 3\% & P3, 7, 9, 10: 0\% & $\mathrm{P} 1,3,4,5,10: 0 \%$ \\
\hline & Max & P10: 97\% & P1, 3, 4: 17\% & P2, 9: 24\% & P1: $14 \%$ & P2, 6, 7: 7\% \\
\hline $\mathrm{E}$ & Max & E10: 93\% & E8: $10 \%$ & E4: $31 \%$ & E3, 4, 5, 6, 7: 3\% & E4: $10 \%$ \\
\hline \multirow{2}{*}{$\mathrm{F}$} & Min & F4: $10 \%$ & F7: 7\% & F2: $7 \%$ & F6, 10: 3\% & F6, 10: 0\% \\
\hline & Max & F10: 59\% & F1: $31 \%$ & F4: $34 \%$ & F2: $28 \%$ & F2, 4: $21 \%$ \\
\hline
\end{tabular}

P physical area, E emotional area, F functional area. 
Early Glottic Carcinoma Treated with High Level of Irradiation

Table 4. Voice handicap index analysis.

\begin{tabular}{|c|c|c|c|c|c|c|c|c|}
\hline Patient & VHI & VD & VHI_P & VD_P & VHI_E & VD_E & VHI_F & VD_F \\
\hline 1 & 11 & Mild & 7 & Mild & 0 & Mild & 4 & Mild \\
\hline 2 & 38 & Moderate & 13 & Moderate & 11 & Moderate & 14 & Moderate \\
\hline 3 & 44 & Moderate & 12 & Moderate & 11 & Moderate & 21 & Severe \\
\hline 4 & 13 & Mild & 2 & Mild & 0 & Mild & 11 & Moderate \\
\hline 5 & 41 & Moderate & 14 & Moderate & 13 & Moderate & 14 & Moderate \\
\hline 6 & 26 & Mild & 7 & Mild & 0 & Mild & 19 & Moderate \\
\hline 7 & 12 & Mild & 0 & Mild & 0 & Mild & 12 & Moderate \\
\hline 8 & 0 & Mild & 0 & Mild & 0 & Mild & 0 & Mild \\
\hline 9 & 2 & Mild & 0 & Mild & 0 & Mild & 2 & Mild \\
\hline 10 & 29 & Mild & 2 & Mild & 8 & Mild & 19 & Moderate \\
\hline 11 & 18 & Mild & 2 & Mild & 0 & Mild & 16 & Moderate \\
\hline 12 & 6 & Mild & 2 & Mild & 0 & Mild & 4 & Mild \\
\hline 13 & 37 & Moderate & 7 & Mild & 13 & Moderate & 17 & Moderate \\
\hline 14 & 41 & Moderate & 9 & Mild & 7 & Mild & 25 & Severe \\
\hline 15 & 49 & Moderate & 11 & Moderate & 8 & Mild & 30 & Severe \\
\hline 16 & 18 & Mild & 0 & Mild & 4 & Mild & 14 & Moderate \\
\hline 17 & 11 & Mild & 3 & Mild & 0 & Mild & 8 & Mild \\
\hline 18 & 2 & Mild & 0 & Mild & 0 & Mild & 2 & Mild \\
\hline 19 & 1 & Mild & 0 & Mild & 0 & Mild & 1 & Mild \\
\hline 20 & 15 & Mild & 7 & Mild & 0 & Mild & 8 & Mild \\
\hline 21 & 37 & Moderate & 11 & Moderate & 5 & Mild & 21 & Severe \\
\hline 22 & 31 & Moderate & 2 & Mild & 7 & Mild & 22 & Severe \\
\hline 23 & 40 & Moderate & 11 & Moderate & 6 & Mild & 23 & Severe \\
\hline 24 & 2 & Mild & 0 & Mild & 0 & Mild & 2 & Mild \\
\hline 25 & 26 & Mild & 9 & Mild & 2 & Mild & 15 & Severe \\
\hline 26 & 92 & Very severe & 30 & Severe & 34 & Very severe & 28 & Severe \\
\hline 27 & 25 & Mild & 9 & Mild & 6 & Mild & 10 & Moderate \\
\hline 28 & 38 & Moderate & 16 & Moderate & 5 & Mild & 17 & Moderate \\
\hline 29 & 1 & Mild & 0 & Mild & 0 & Mild & 1 & MILD \\
\hline
\end{tabular}

VHI: Voice Handicap Index, VD: Vocal Disability,_P: physical area,_E: emotional area,_F: functional area.

[4,11-14,18,21]. For T1-2 N0 glottic cancer, hypofractionated radiation is usually preferred, administering a total dose of 63 Gy at $2.25 \mathrm{~Gy} /$ fraction per day, 5 days/ week [23]. If $2 \mathrm{~Gy} /$ fraction is used, a total dose $>66 \mathrm{~Gy}$ is commonly recommended. Lee et al. [24] noted that in the Tis/T1 squamous cell carcinomas of the glottic subgroup, doses $\geq 66$ Gy were associated with significantly higher local control (versus doses < 66 Gy).

Confident of improved local control is achieved with doses $\geq 66$ Gy (with conventional use of 2 Gy fractions), we have used a total dose of $70 \mathrm{~Gy}$ at $200 \mathrm{cGy} /$ fraction to the PTV. Comparing hypofractionation and conventional treatment regimens, the biological equivalent dose valuation shows how a total dose of 63 Gy with 2.25 Gy/frac- 
tion $(\alpha / \beta$ tumor $=10)$ is equivalent to a dose of $64.31 \mathrm{~Gy}$, while a total dose of $60 \mathrm{~Gy}$ at $2.4 \mathrm{~Gy} /$ fraction is equivalent to 62 Gy in 2 Gy daily fractions. In our series, because of the higher dose, there was the possibility of poor voice quality. Whereas VHI index analysis shown a voice disability even inferior to that reported in literature $[13,14,18]$.

Peeters et al. [13] compared voice quality in a cohort of patients treated by radiotherapy or endoscopic laser surgery for T1a glottic carcinoma. The results were worse in patients treated with radiation, using 50 - 70 Gy in 20 - 35 daily fractions: a mild vocal disability was reported in $40 \%$ of these patients. Jepsen et al. [14] describe voice outcome, using VHI index, in patients treated for laryngeal cancer, with or without adjuvant radiotherapy. The average VHI of patients treated for glottic cancer was 42.2. Levendag et al. [18] reported a VHI of 12.7 in 164 patients with T1a glottic cancer, using a 4D conformal technique for single vocal cord irradiation. Di Nicola et al. [19] considered a total of 40 patients with T1 squamous cell carcinoma of the true vocal cord. 18 patients were treated with $66 \mathrm{~Gy}$ in 33 daily fractions of 2 Gy and 22 patients received a total dose of 60 Gy in 25 fractions of $2.4 \mathrm{~Gy}$. The average VHI 1 year after the end of radiotherapy was 23.2 (SD 12.4) and no significant difference between the two arms was documented.

Acute reactions to radiotherapy were mild and were managed with topical anesthetics, anti-inflammatory agents and moisturizer. Despite the high level of irradiation (70 Gy), treatment was well tolerated. No patients resulted in a treatment break. Toxicity was assessed according to Common Toxicity Criteria (Version 2.0): highest frequency of dysphagia G2 (18 patients) was observed during III week of treatment, while highest frequency of erythema G1 occurred in V and VI week (6 and 7 patients respectively). Four patients revealed dysphonia during $\mathrm{V}$ week and it persisted for 4 - 5 weeks. Toxicity late effects, such as laringeal edema, were not observed, probably considering fraction size: all patients received 70 Gy in 7 weeks at 200 cGy/fraction. The incidence of laringeal edema increases with greater total dose, field size, dose per fraction and $\mathrm{T}$ stage [25]. In several studies $[26,27]$ the incidence of severe late complications was higher in patients who received greater than 2.25 Gy per fraction.

\section{Conclusions}

Therapy outcome has been satisfactory in terms of overall survival, disease free survival, voice quality and toxicity. No deaths occurred during follow-up (mean 33.5 months) and local recurrence occurred in two patients only.

With regard to VHI, questionnaire selection proved to be effective as we gathered consistent answers, we covered three areas of interest (physical, emotional, functional) and we improved our relationship with each patient. Our statistical analysis, resulted in a slightly better VHI than average data found in recent literature.

Therefore we confirm that radiotherapy produces acceptable functional results, with limited repercussion on patient's life, as evidenced by the VHI. We did not observe any severe toxicity and we only reported mild reactions (few cases of dysphagia, dysphonia and erythema) despite a total dose of $70 \mathrm{~Gy}$.

\section{REFERENCES}

[1] R. Siegel, E. Ward, O. Brawley and A. Jemal, "Cancer Statistics,” CA: A Cancer Journal for Clinicians, Vol. 63, No. 1, 2013, pp. 11-30. http://dx.doi.org/10.3322/caac.21166

[2] L. P. Bron, D. Soldati, M. L. Monod, C. Mégevand, E. Brossard, P. Monnier and P. Pasche, "Treatment of Early Stage Squamous Cell Carcinoma of the Glottis Larynx: Endoscopic Surgery or Cricohyoidoepiglottopexy versus Radiotherapy," Head \& Neck, Vol. 23, No. 10, 2001, pp. 823-829. http://dx.doi.org/10.1002/hed.1120

[3] P. Dey, D. Arnold, R. Wight, K. Mackenzie, C. Kelly and J. Wilson, "Radiotherapy versus Open Surgery versus Endolaryngeal Surgery (with or without Laser) for Early Laryngeal Squamous Cell Cancer," Cochrane Database of Systematic Reviews, Vol. 2, 2002, Article ID: CD002027.

[4] C. van Gogh, I. M. Verdonck-de Leeuw, B. Boon-Kamma, J. Langendijk, D. Kuik and H. Mahieu, “A Screening Questionnaire for Voice Problems after Treatment of Early Glottic Cancer,” International Journal of Radiation Oncology, Biology, Physics, Vol. 62, No. 3, 2005, pp. 700-705. http://dx.doi.org/10.1016/j.jjrobp.2004.10.027

[5] A. Gallo, M. de Vincentiis, V. Manciocco, M. Simonelli, M. L. Fiorella and J. Shah, " $\mathrm{CO}_{2}$ Laser Cordectomy for Early-Stage Glottic Carcinoma: A Long-Term Follow-Up of 156 Cases,” Laryngoscope, Vol. 112, No. 2, 2002, pp. 370-374. http://dx.doi.org/10.1097/00005537-200202000-00030

[6] J. C. Smith, J. T. Johnson, D. M. Cognetti, D. P. Landsittel, W. E. Gooding, E. R. Cano and E. N. Myers, “Quality of Life, Functional Outcome, and Costs of Early Glottic Cancer,” Laryngoscope, Vol. 113, No. 1, 2003, pp. 68-76. http://dx.doi.org/10.1097/00005537-200301000-00013

[7] J. H. Brandenburg, "Laser Cordotomy versus Radiotherapy: An Objective Cost Analysis,” Annals of Otology, Rhinology, and Laryngology, Vol. 110, No. 4, 2001, pp. 312-318.

[8] F. N. Batalla, M. J. Caminero Cueva, B. Gonzàlez, J. L. Pendàs, C. G. Gil, A. L. Llames, R. A. Pantiga and C. S. Nieto, "Voice Quality after Endoscopic Laser Surgery and Radiotherapy for Early Glottic Cancer: Objective Measurements Emphasizing the Voice Handicap Index,” European Archives of Oto-Rhino-Laryngology, Vol. 265, 
No. 5, 2008, pp. 543-548.

http://dx.doi.org/10.1007/s00405-007-0512-9

[9] A. Mlynarek, K. Kost and R. Gesser, "Radiotherapy versus Surgery for Early T1-T2 Glottic Carcinoma,” Journal of Otolaryngology, Vol. 35, No. 6, 2006, pp. 413-419. http://dx.doi.org/10.2310/7070.2005.0036

[10] A. S. Jones, B. Fish, J. E. Fenton and D. J. Husband, “The Treatment of Early Laryngeal Cancer (T1-T2 N0): Surgery or Irradiation?” Head \& Neck, Vol. 26, No. 2, 2004, pp. 127-135. http://dx.doi.org/10.1002/hed.10361

[11] I. M. Verdonck-de Leeuw, R. B. Keus, F. J. Hilgers, F. J. Koopmans-van Beinum, A. J. Greven, J. M. de Jong, G. Vreeburg and H. Bartelink, "Consequences of Voice Impairment in Daily Life for Patients Following Radiotherapy for Early Glottis Cancer: Voice Quality, Vocal Function, and Vocal Performance," International Journal of Radiation Oncology, Biology, Physics, Vol. 44, No. 5, 1999, pp. 1071-1078. http://dx.doi.org/10.1016/S0360-3016(99)00110-8

[12] C. M. Waghmare, J. Agarwal and G. K. Bachher, "Quality of Voice after Radiotherapy in Early Vocal Cord Cancer," Expert Review of Anticancer Therapy, Vol. 10, No. 9, 2010, pp. 1381-1388. http://dx.doi.org/10.1586/era.10.126

[13] A. J. Peeters, C. van Gogh, K. M. Goor, I. M. Verdonckde Leeuw, J. A. Langendijk and H. F. Mahieu, "Health Status and Voice Outcome after Treatment for T1a Glottica Carcinoma," European Archives of Oto-Rhino-Laryngology, Vol. 261, No. 10, 2004, pp. 534-540. http://dx.doi.org/10.1007/s00405-003-0697-5

[14] M. C. Jepsen, D. Gurushanthaiah, N. Roy, M. E. Smith, S. D. Gray and R. K. Davis, "Voice, Speech, and Swallowing Outcomes in Laser-Treated Laryngeal Cancer," Laryngoscope, Vol. 113, No. 6, 2003, pp. 923-928. http://dx.doi.org/10.1097/00005537-200306000-00001

[15] S. J. Stoeckli, M. Guidicelli, A. Schneider, A. Huber and S. Schmid, "Quality of Life after Treatment for Early Laryngeal Carcinoma," European Archives of Oto-RhinoLaryngology, Vol. 258, No. 2, 2001, pp. 96-99. http://dx.doi.org/10.1007/s004050000307

[16] M. van der Torn, I. M. Verdonck-de Leeuw, D. J. Kuik and H. F. Mahieu, "Communicaive Suitability of Voice Following Radiotherapy for T1 Glottic Carcinoma: Testing the Reliability of a Rating Instrument," Journal of Voice, Vol. 16, No. 3, 2002, pp. 398-407. http://dx.doi.org/10.1016/S0892-1997(02)00111-X

[17] S. M. Cohen, C. G. Garret, W. D. Dupont, R. H. Ossoff and M. S. Courey, "Voice-Related Quality of Life in T1 Glottic Cancer: Irradiation versus Endoscopic Excision," Annals of Otology, Rhinology, and Laryngology, Vol. 115, No. 8, 2006, pp. 581-586.

[18] P. C. Levendag, D. N. Teguh, F. Keskin-Cambay, A. AlMamgani, P. V. Rooij, E. Astreinidou, S. L. Kwa, B. Heijmen, D. A. Monserez and S. Osman, "Single Vocal Cord Irradiation: A Competitive Treatment Strategy in Early Glottic Cancer,” Radiotherapy \& Oncology, Vol. 101, No. 3, 2011, pp. 415-419. http://dx.doi.org/10.1016/j.radonc.2011.05.026

[19] L. Di Nicola, G. L. Gravina, F. Marampon, P. Bonfili, S. Buonopane, M. Di Staso, C. Festuccia, P. Franzese, M. Tombolini and V. Tombolini, "The Impact of Conventional or Hypofractionated Radiotherapy on Voice Quality and Oncological Outcome in Patients with Early Glottic Cancer,” Oncology Reports, Vol. 24, No. 5, 2010, pp. 1383-1388.

[20] A. Schindler, F. Ottaviani, F. Mozzanica, C. Bachmann, E. Favero, I. Schettino and G. Ruoppolo, "Cross-Cultural Adaptation and Validation of the Voice Handicap Index into Italian,” Journal of Voice, Vol. 24, No. 6, 2010, pp. 708-714. http://dx.doi.org/10.1016/j.jvoice.2009.05.006

[21] I. Hocevar-Boltezar, M. Zargi and I. Honocodeevar-Boltezar, "Voice Quality after Radiation Therapy for Early Glottis Cancer," Archives of Otolaryngology-Head and Neck Surgery, Vol. 126, No. 9, 2000, pp. 1097-1100. http://dx.doi.org/10.1001/archotol.126.9.1097

[22] I. Honocodeevar-Boltezar and M. Zargi, "Voice Quality after Radiation Therapy for Early Glottic Cancer," Archives of Otolaryngology_Head and Neck Surgery, Vol. 126, No. 9, 2000, pp. 1097-1110. http://dx.doi.org/10.1001/archotol.126.9.1097

[23] H. Yamazaki, K. Nishiyama, E. Tanaka, M. Koizumi and M. Chatani, "Radiotherapy for Early Glottic Carcinoma (T1N0M0): Results of Prospective Randomized Study of Radiation Fraction Size and Overall Treatment Time," International Journal of Radiation Oncology, Biology, Physics, Vol. 64, No. 1, 2006, pp. 77-82. http://dx.doi.org/10.1016/j.ijrobp.2005.06.014

[24] J. Lee, M. Machtay, M. McKenna, G. Weinstein, D. Markiewicz, R. Krisch and M. Kligerman, "Radiotherapy with 6-Megavolt Photons for Early Glottic Carcinoma: Potential Impact of Extension to the Posterior Vocal Cord,” American Journal of Otolaryngology, Vol. 22, No. 1, 2001, pp. 43-54. http://dx.doi.org/10.1053/ajot.2001.20679

[25] K. A. Dinshaw, V. Sharma, J. P. Agarwal, S. Ghosh and R. Havaldar, "Radiation Therapy in T1-T2 Glottic Carcinoma: Influence of Various Treatment Parameters on Local Control/Complications," International Journal of Radiation Oncology, Biology, Physics, Vol. 48, No. 3, 2000, pp. 723-735. http://dx.doi.org/10.1016/S0360-3016(00)00635-0

[26] S. M. Deore, S. J. Supe, V. Sharma and K. A. Dinshaw, "The Predictive Role of Bioeffect Dose Models in Radiation-Induced Late Effects in Glottic Cancers," International Journal of Radiation Oncology, Biology, Physics, Vol. 23, No. 2, 1992, pp. 281-284.

http://dx.doi.org/10.1016/0360-3016(92)90742-Z

[27] W. M. Mendenhall, R. J. Amdur, C. G. Morris and R. W. Hinerman, "T1-T2N0 Squamous Cell Carcinoma of the Glottic Larynx Treated with Radiation Therapy,” Journal of Clinical Oncology, Vol. 19, No. 20, 2001, pp. 40294036. 